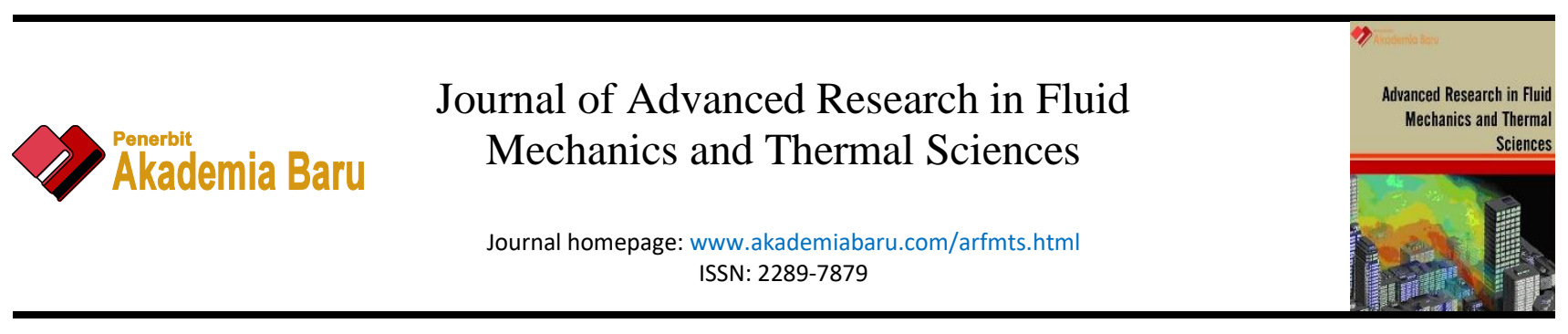

\title{
Effect of Multi Size Porous Media Properties on the Flow of Non-Darcy Regime
}

\author{
Asaad Salim Bded ${ }^{1,}$, , Farhan Lafta Rashid ${ }^{1}$ \\ 1 Department of Petroleum Engineering, University of Kerbala, Iraq
}

\section{ARTICLE INFO \\ ABSTRACT}

\section{Article history:}

Received 11 December 2019

Received in revised form 11 January 2020

Accepted 12 January 2020

Available online 25 March 2020

Keywords:

Flow in porous media; permeability; Forchheimer number; non-Darcy Regime
The objective of this paper is to study effect of multi size media on behavior of fluid at post-Darcy flow regime as porous medias differences in the sizes and weight. Crude oil used as fluid within three types of medias (gravels, glass balls, SiLibeads), and Kozeny -Carman model is used to estimate the head loss experimentally under changing on the flow rate. There are two coefficients have effect on non-Darcy flow regime (Reynolds number, Forchheimer number) at the same time depends it's on properties of media; one of them is determined by using linear regression model to predicts the Forchheimer numbers. The permeability, porosity and Tortuosity are determined theoretically and all of them depend on properties of fluid and media. the test result shows the theoretical analysis from the Forchheimer number is directly proportional with effect of post-Darcy flow regime, and that non-Darcy behavior has good value with glass media ( 0.5166 porosity) .Porous media properties shows the permeability and non-Darcy coefficient have effect on non-Darcy behavior and inertia coefficient depends on porosity and properties of permeable bed.

\section{Introduction}

A property of multi size of material as porous medium have effects on the regime of flow and is one of application reservoir engineering, packed beds in petroleum industries and can be used in wide variety of engineering applications [1]. The fluid behavior inside porous material changing with material properties in three zones start from pre-Darcy zone then Darcy zone and post-Darcy zone at turbulent flow. The types of flow occurring according to how fast the fluid is moving relative to how viscous it is, properties of material have effects on the flow of behavior for different particle size and type of fluid. The studies illustrate uses in the porous media as in, Bded [2] who studied the gas flow performance through porous media, and as a result the gas flow rate was an important parameter influencing bed for single size of backing.

\footnotetext{
* Corresponding author.

E-mail address: asaadsalim78@gmail.com (Asaad Salim Bded)
} 
Some of parameters have effects on the post-Darcy zone behavior in porous media, so the known them is very important [3]. researcher studied the flow through granular materials using different rounded aggregates sizes and hydraulic gradients in order to assess the fluid flow behavior at high velocity, the media characteristics with high porosity values and large pores, for such conditions; the linear theories applicability to characterize flow of water has been calculated comprehensively. So, he studied the non-Darcy flow coefficients with increase in grain diameter, their results are valid for uniform materials only. Mohammed N. Abbas [4] developed an equation of porosity as a function of parameters affecting the water flow porosity during medium bed of spherical particles for uniform permeable media.

When the flow be turbulent (post-Darcy flow) the regime of flow be non-Darcy, and there are parameters having effects on the non- Darcy and this clear in some of studies like Jan H. Roy Snoeijers [5]. Their studies gave new experimental information for non-flow behavior in different porous materials changing from medium sands to gravel. Their studies show that fluid flow resistance in permeable media can be predicted by the pore structure geometry and the equations relate the Forchheimer parameters to the porosity and characteristic pore length. This study shows that the geometry of the pore comes from the uniform size and non-uniform particle size and leads to change in the path of flow from linear to nonlinear. Wojciech S., Anna T. [6] studied some of flow parameters such as permeability, Forchheimer coefficient, based on pressure drop and flow rate for flow rates measurements exceed the linear Darcy's flow regime. The results showed that a greater work dedicated to simulate the fluid flow through porous media with specific benefit in granular beds. Hidayah et al., [7] showed that the porosity is an important factor in blocks performance. Razali and Sadikin [8] studied the prediction of flow in the microchannel and gives offered advantages in using microchannel based on pressure and velocity of fluid through porous media. Kumar [9] suggest the relation equivalent for Reynolds number with Forchheimer number to show the flow system at different points. and find Forchheimer number takes into smooth change of flow in two zone from transition and inertia regimes.

From previous studies we can conclude the problem in this study by divided the regimes of flow at four sections started from Darcy flow and throughout to the section of Forchheimer flow to reach for the nonlinear relation under turbulent flow (non-Darcy flow) proportional to the velocity square, this will help us to determine the purpose of this study.

The aim of research is to show the effect of multi particle size to shape uniform and non-uniform from porous media (gravels, glass bed, SiLibeads) by viscose fluid (crude oil) on the non-Darcy behavior regime, and effect of Properties system on its behavior.

\section{Regimes of Flow in The Permeable Media}

Many of different flow regimes can be distinguished for the flow in porous media. Started from Darcy flow zone behavior for dominant of viscous forces with linear pressure gradient as in Forchheimer suggestion [10]. The second zone after increasing Reynolds number is Forchheimer flow (transition zone), this flow had effect from laminar inertia flow and the inertial forces to give nonlinear relation between pressure gradient and Darcy velocity. The third zone is turbulent flow (non-Darcy flow) which is highly unstable and disorder flow system at high Reynolds number and dominated by eddies and the inertial forces become strong, pressure gradient is proportional to the velocity square $[10,11]$.

Forchheimer suggested the flow within porous media in the below form and can collect all three zones in the following equation: 
$\frac{\Delta p}{L}=\frac{\mu u}{k}+\rho k_{F} u^{2}=\alpha u+\beta u^{2}$

The pressure gradient in the zone of porous media can be characterized by several factors, as in the Eq. (2). In general:

$$
(\Delta p)_{\text {total }}=(\Delta p)_{\text {viscous }}+(\Delta p)_{\text {inertial }}
$$

In this study the third zone is important and non-Darcy behavior occurs at it, so the assumptions of Eq. (1) identify the two criterions of Reynolds number and the Forchheimer number. Forchheimer number has related to the effect of non-Darcy. It equals the ratio of pressure gradient to viscous resistance [12].

\section{Crude Oil and Media Properties}

\subsection{Properties of Iraqi Crude Oil}

The crude oil used as fluid in this research and all physical properties are tested in lab of petroleum properties in Petroleum Engineering Department (University of Kerbala) as shown in Table 1.

\begin{tabular}{|c|c|c|}
\hline Physical properties & Value & Method of test \\
\hline Density (gm/ml) & 0.693 & $\begin{array}{l}\text { Pycnometer } \\
\text { method ASTM- } \\
\text { D-1217 }\end{array}$ \\
\hline $\begin{array}{l}\text { Kinematic viscosity } \\
\left(\mathrm{m}^{2} / \mathrm{s}\right)\end{array}$ & $\begin{array}{l}8.64045 \\
* 10^{-6}\end{array}$ & $\begin{array}{l}\text { viscometer } \\
\text { method ASTM }\end{array}$ \\
\hline $\begin{array}{l}\text { Dynamic viscosity } \\
\text { (kg/m.s) }\end{array}$ & 0.005988 & D-445 \\
\hline
\end{tabular}

\subsection{Media Properties}

Three type of material used as porous media for different size and shape, two of them uniform in particle size and one non uniform in the size and shape (Gravels, Glass bed, SiLibeads) as shown in Figure 1. The permeability, porosity and Tortuosity are determined theoretically and experimentally and all of them depend on properties of media for each one, as shown in Table 2.

Table 2

Properties of three types of media

\begin{tabular}{llll}
\hline Type of medium & $\begin{array}{l}\text { Diameter } \\
(\mathrm{mm})\end{array}$ & $\begin{array}{l}\text { Weight } \\
(\mathrm{gm})\end{array}$ & Porosity \\
\hline Gravels & 14.86421 & 425.23 & 0.407605 \\
Glass balls & 20 & 378.01 & 0.5166 \\
SiLibeads & 1.65 & 500 & 0.3866 \\
\hline
\end{tabular}



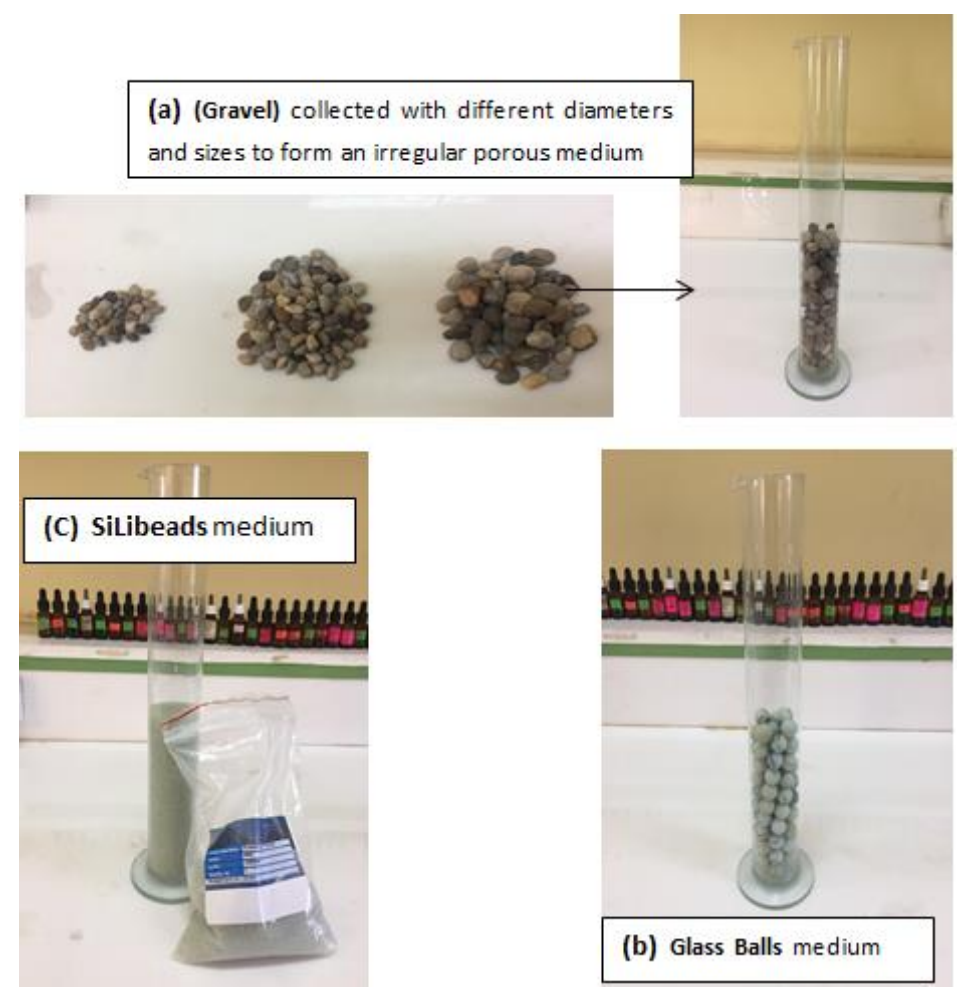

Fig. 1. Type of material used as porous medium

\section{Experimental and Theoretical Procedures}

\subsection{Experimental Part}

In this paper we have relied on the experiments that available in the fluid laboratory in Petroleum Engineering Department as a source to conduct our own calculations, the following device was used in the laboratory, as shown in Figure 2.

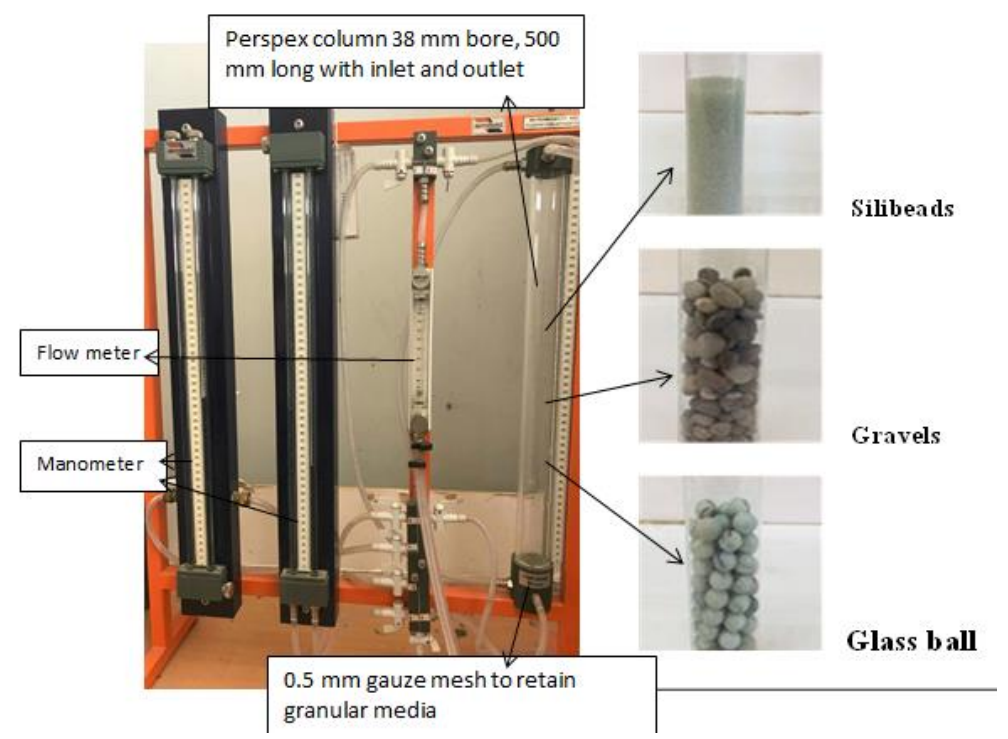

Fig. 2. The experimental Rig

To prepare the porous media, it should be determine the porosity and permeability, the first material is (SiLibeads) well rounded quartz grains sand have the density of sand equal to $2650 \mathrm{~kg} / \mathrm{m} 3$ with mass of sand equal to $0.54 \mathrm{~kg}$, the level of the media (permeable bed) length equal to $300 \mathrm{~mm}$ 
for all three type putted in cylindrical with diameter equal to $38 \mathrm{~mm}$. we can determine the porosity $(\varphi)$ for each media from the following Eq. (3) [13]:

$\phi=\frac{\text { Volume of voids in a bed }}{\text { Total volume(voids and solids) }}$

For the second media, the gravel was collected with different diameters and sizes to form an irregular porous media to study the effect of regularity of porous medium on the nature and characteristics of the flow. Then divided it into three sections as shown in Figure 2. To compute the equivalent diameter for the gravel media, Eq. (4) used to find equivalent diameter:

$d_{e f f}=\frac{1}{\sum_{i=1}^{n} \frac{x_{i}}{d_{i}}}$

where: $x_{i}=$ particle fractional weight, $d_{i}=$ particle diameter

The third media is a glass balls of equal diameter to form a regular porous media having the diameter of $20 \mathrm{~mm}$ with weight equal to $378.01 \mathrm{gm}$ at same length of bed ( $L=300 \mathrm{~mm}$ ). For all types of media weighted by a sensitive balance.

For different flow rate $(Q)$, read on the flow meter, the readings increasing from laminar to turbulent flow, the crude oil initially passes up through the porous bed grains, under these condition Darcy's law and Kozeny-Carman equation applies to determine the head loss or pressure drop from Eq. (5):

$\frac{\mathrm{dh}}{\mathrm{dL}}=\frac{5 \mu \mathrm{Vs}}{\rho \mathrm{g}} \frac{(1-\emptyset)^{2}}{\emptyset^{3}}\left(\frac{6}{\mathrm{~d}}\right)^{2}$

Then under each condition, can be calculate Reynolds number from Eq. (6):

$\operatorname{Re}=\frac{\rho \mathrm{udp}}{\mu}$

The pressure gradient should be calculated and Reynolds number will help us to determine the boundary of non-Darcy zone.

\subsection{Theoretical Part}

Forchheimer number can be defined as the ratio of interaction liquid with solid pressure gradient to the viscous resistance [1]:

Forchheimer number $=\frac{k \beta \rho u}{\mu}$

This is coming from Eq. (1), in which the right-hand side $(\mu \mathrm{u} / \mathrm{k})$ is viscous resistance and the term $(\beta$ $\rho u^{2}$ ) liquid/solid interactions [1], so the determination of the Forchheimer number needed to the permeability and non-Darcy coefficient also to crude oil physical properties. Can get these parameters from the Darcy Eq. (1) after rewritten as: 
$\frac{\Delta \mathrm{p}}{\mathrm{L} \mu \mathrm{u}}=\frac{1}{\mathrm{k}}+\beta \frac{\rho \mathrm{u}}{\mu}$

where: $\beta=$ non-Darcy coefficient, $\mathrm{k}=$ permeability

By applying one of the numerical methods which known by (Linear Regression Model), so Eq. (8) should be linearized to find the value of $k$ and $\beta$ to get Eq. (9).

$\mathrm{Y}=\frac{1}{\mathrm{k}}+\beta \mathrm{X}$

Eq. (8) became linear equation and easy to find the value of slope $\beta$ and intersection $1 / k$ at different flow rates, and may be applied to all types of porous medias $[1,14]$.

\subsection{Tortuosity Determination}

Tortuosity is calculated from the tortuosity and porosity relationship for a permeable bed with mixed in particles depends on packing preparation applied:

Tortuosity $=\frac{1}{\phi^{n}}$

where $(n)$ represents a numerical value, and it ranges from 0.4 to 0.5 for bed of loose permeable and dense permeable, respectively $[13,15]$.

\section{Results and Discussion}

\subsection{Effect of Media Properties on Post-Darcy Flow}

From theoretical analysis of permeability and post Darcy (non-Darcy) coefficient by using Linear Regression Model with Eq. (8), the value of slope $\beta$ and intersection $1 / k$ are determined as shown in Figure 3(a) and Figure 3(b) for gravels and glass balls material, but in SiLibeads material there is no boundary of non-Darcy zone. Because of the linear relationship between head loss and flow rate, this is deal with $[10,11]$. Table 3 illustrate the permeability and non-Darcy coefficient values for each type of media, viscous resistance in the gravels more than glass balls material and SiLibeads, because of porosity of each one, but at Inertial Resistance (non-Darcy coefficient) for gravels material have value equal to 1040 at porosity 0.407 more than glass material at porosity 0.51 , and at porosity 0.3866 the non-Darcy coefficient was 238.034 .

Table 3

Permeability and non-Darcy coefficient values

\begin{tabular}{|c|c|c|c|}
\hline $\begin{array}{l}\text { Type of porous } \\
\text { media }\end{array}$ & $\begin{array}{l}\text { Viscous } \\
\text { Resistance } \\
\text { Permeability, } \mathrm{k}, \\
\mathrm{m}^{2}\end{array}$ & $\begin{array}{l}\text { Inertial } \\
\text { Resistance Non- } \\
\text { Darcy coefficient, } \\
\beta, \mathrm{m}^{-1}\end{array}$ & Porosity, $\phi$ \\
\hline Gravels & $8 * 10^{-7}$ & 1040 & 0.407605 \\
\hline Glass balls & $6 * 10^{-7}$ & 306.8 & 0.5166 \\
\hline SiLibeads & $1.55157 * 10^{-6}$ & 238.0399 & 0.3866 \\
\hline
\end{tabular}




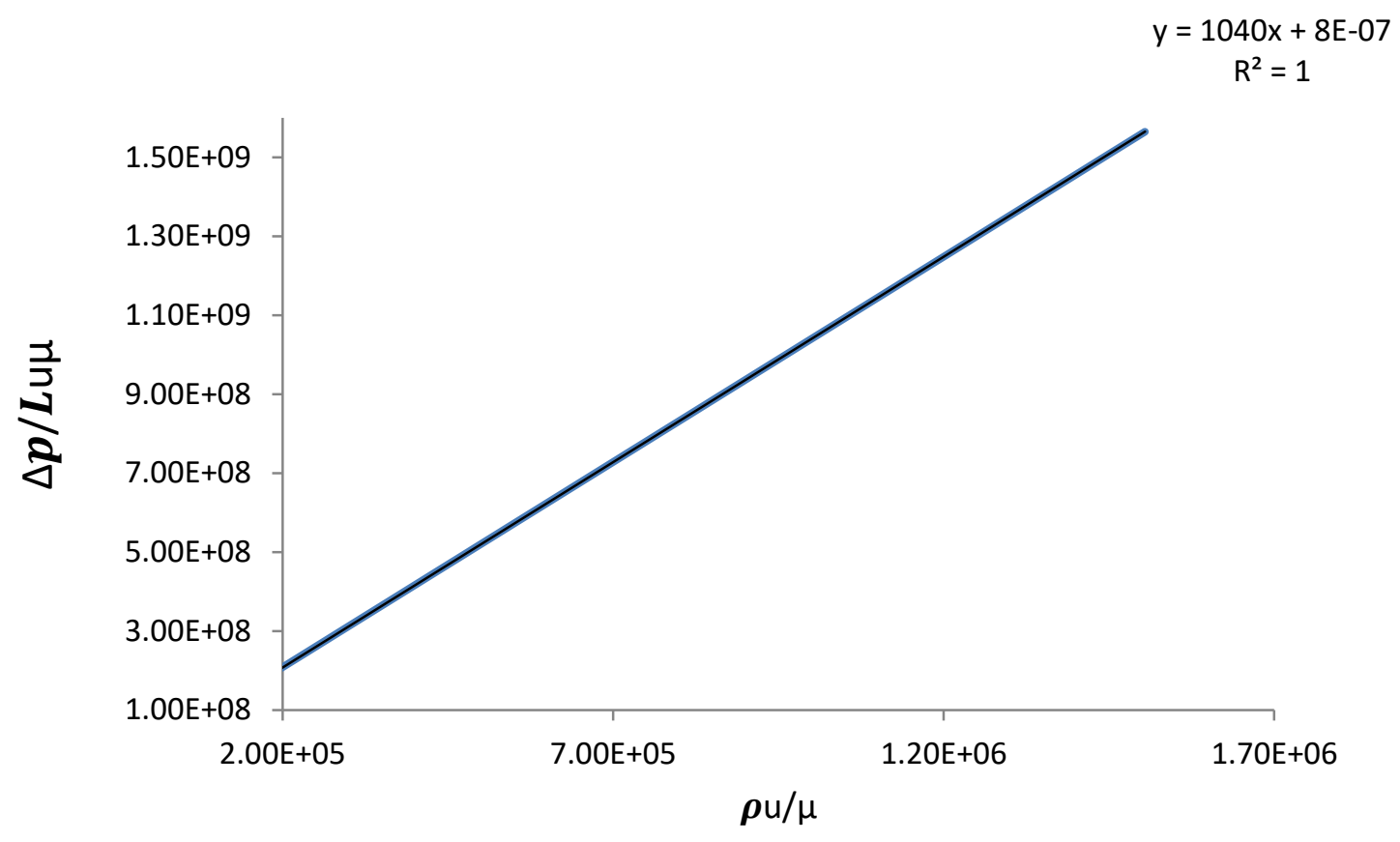

(a) Graval medium

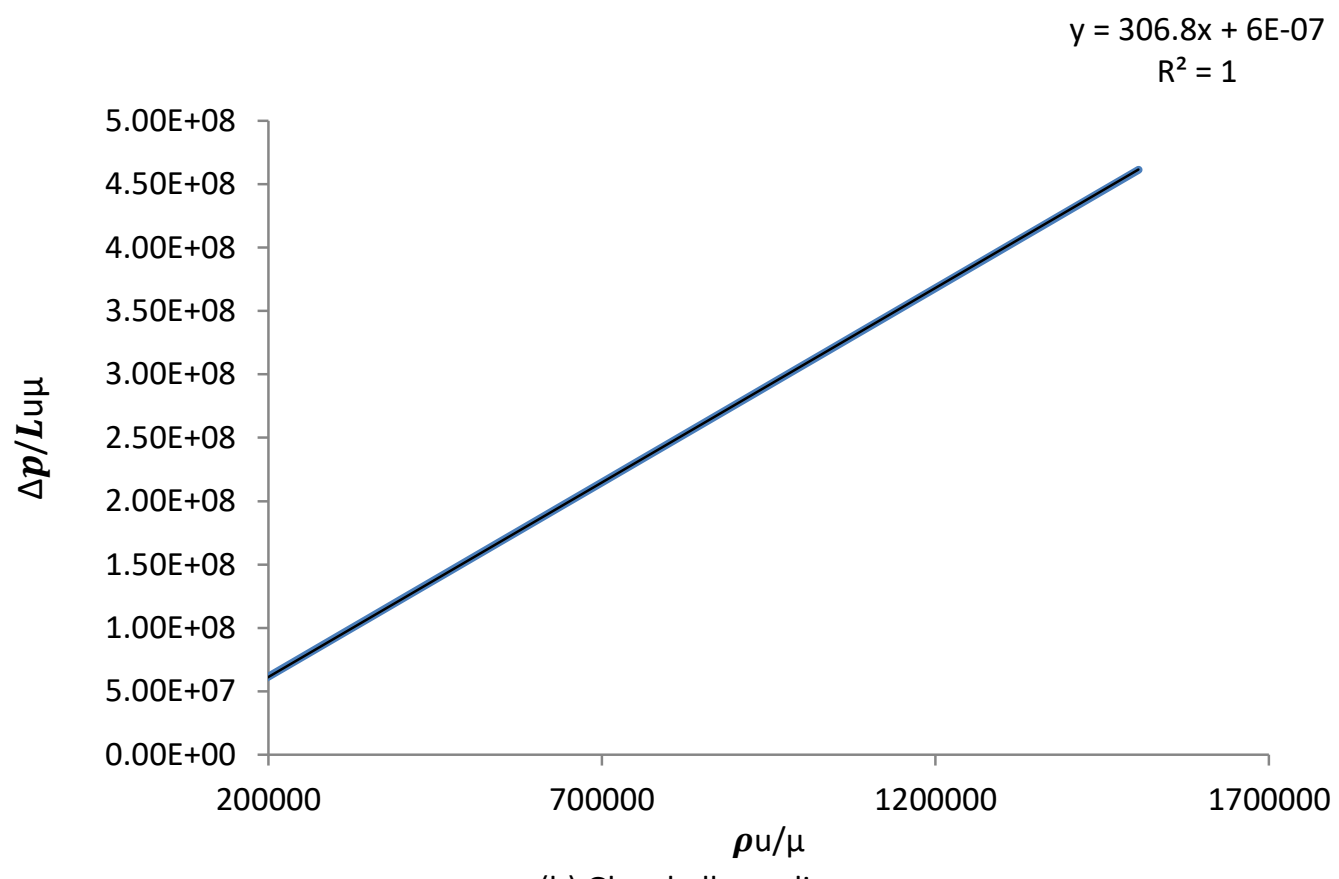

(b) Glass balls medium

Fig. 3. Prediction of coefficient of post-Darcy and permeability, (a) Gravels, (b) Glass material

\subsection{Criterion of Forchheimer Number for The Flow in Post-Darcy Regime by Multi Porous edia}

By applying Eq. (7) the Forchheimer number will determine point of effect on the non-Darcy in three porous media as shown in Table 4 which is illustrate Forchheimer number in the gravel particles, glass particles and SiLibeads at different flow rate. Figure 4 shows the relation between non-Darcy flow and velocity in three porous media, Forchheimer number takes place at progressive 
and smooth replacing in the flow zone between transition and inertia resistance regimes, this point deals with suggestion of Kumar [9], so it is more clear from Figure 4 that the Forchheimer number have directly effect on Post-Darcy (non-Darcy) as on Z. Zeng [1]. That means the non-Darcy effect is more acute in low value of permeable porous media.

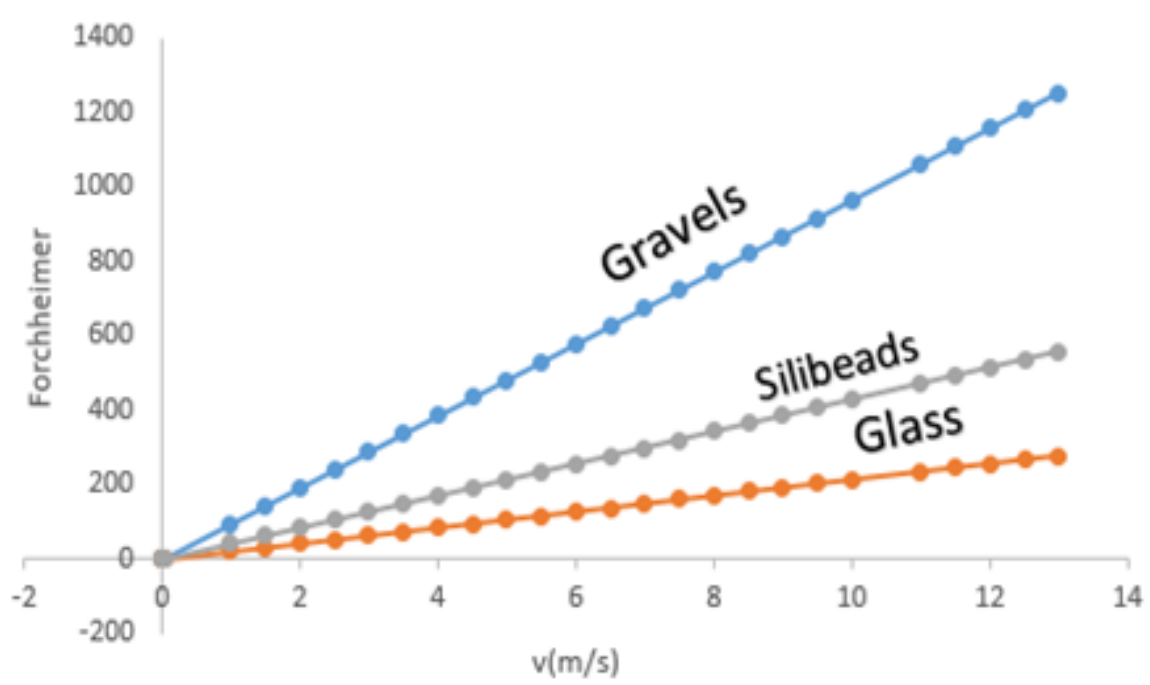

Fig. 4. Illustrate relation between Forchheimer number and flow rate (velocity $\mathrm{m} / \mathrm{s}$ )

\section{Table 4}

Values of Forchheimer number at different flow rate (velocity $\mathrm{m} / \mathrm{s}$ ) in Gravel particles, glass particles and SiLibeads

\begin{tabular}{llll}
\hline $\mathrm{v}(\mathrm{m} / \mathrm{s})$ & \multicolumn{2}{l}{ Forchheimer Number } & \\
& Gravel Particles & Glass Particles & SiLibeads \\
\hline 0.0001 & 0.009642 & 0.002133 & 0.00428 \\
0.001 & 0.096417 & 0.021332 & 0.0428 \\
0.01 & 0.96417 & 0.21332 & 0.428 \\
0.05 & 4.82085 & 1.0666 & 2.14 \\
1 & 96.417 & 21.332 & 42.8 \\
1.5 & 144.6255 & 31.998 & 64.2 \\
2 & 192.834 & 42.664 & 85.6 \\
2.5 & 241.0425 & 53.33 & 107 \\
3 & 289.251 & 63.996 & 128.4 \\
3.5 & 337.4595 & 74.662 & 149.8 \\
4 & 385.668 & 85.328 & 171.2 \\
4.5 & 433.8765 & 95.994 & 192.6 \\
5 & 482.085 & 106.66 & 214 \\
5.5 & 530.2935 & 117.326 & 235.4 \\
6 & 578.502 & 127.992 & 256.8 \\
6.5 & 626.7105 & 138.658 & 278.2 \\
7 & 674.919 & 149.324 & 299.6 \\
7.5 & 723.1275 & 159.99 & 321 \\
8 & 771.336 & 170.656 & 342.4 \\
8.5 & 819.5445 & 181.322 & 363.8 \\
9 & 867.753 & 191.988 & 385.2 \\
9.5 & 915.9615 & 202.654 & 406.6 \\
10 & 964.17 & 213.32 & 428 \\
11 & 1060.587 & 234.652 & 470.8 \\
\hline
\end{tabular}




\subsection{Effect of Permeable Bed Properties on Tortuosity Factor}

The tortuosity of sphere material can be considered as a main parameter affecting on the flow within different porous media. Table 5 shows the value of tortuosity dependence on porosity from Eq. (10) for three porous materials, and we can see in the uniform media which give us the value of tortuosity dependent on the dense permeable bed same of study [4], but at the gravel media, the value of tortuosity was more than SiLibeads and glass balls material, because of non-uniform shape of the gravel media, that is mean that the inertia coefficient depends on porosity and properties of permeable bed.

\begin{tabular}{|c|c|c|}
\hline \multicolumn{3}{|c|}{$\begin{array}{l}\text { The tortuosity and porosity of three porous } \\
\text { materials }\end{array}$} \\
\hline $\begin{array}{l}\text { Type of porous } \\
\text { media }\end{array}$ & Porosity & Tortuosity \\
\hline Gravels & 0.407605 & 1.566 \\
\hline Glass balls & 0.5166 & 1.391 \\
\hline SiLibeads & 0.3866 & 1.4626 \\
\hline
\end{tabular}

\section{Conclusions}

Effect of multi size porous media properties on the flow of non-Darcy regime was studied at three type of material (gravels, glass balls, SiLibeads) with change in flow rate of crude oil through porous media.

Three zone appears in medias changing from linear equation to nonlinear between flow rate and pressure gradient, this help us to determine the non-Darcy flow and point of start for Forchheimer number. Forchheimer number takes place in the flow zone between transition and inertia resistance regimes after changing in flow rate, so it is more clear that the Forchheimer number have directly effect on Post-Darcy (non-Darcy), that means the non-Darcy effect is more acute in low value of permeable porous media and at the same time the properties of multi size have effect on the tortuosity factor.

\section{References}

[1] Zeng, Zhengwen, and Reid Grigg. "A criterion for non-Darcy flow in porous media." Transport in Porous Media 63, no. 1 (2006): 57-69. https://doi.org/10.1007/s11242-005-2720-3

[2] Bded, Asaad Salim. "Performance Simulation for Gas Flow through a Porous Media in Packed bed Columns using CFD." In IOP Conference Series: Materials Science and Engineering, vol. 579, no. 1, p. 012001. IOP Publishing, 2019. https://doi.org/10.1088/1757-899X/579/1/012001

[3] Sedghi-Asl, Mohammad, and Hassan Rahimi. "Adoption of Manning's equation to 1D non-Darcy flow problems." Journal of Hydraulic Research 49, no. 6 (2011): 814-817. https://doi.org/10.1080/00221686.2011.629911

[4] Abbas, Mohammed Nasif. "Modeling of porosity equation for water flow through packed bed of monosize spherical packing." Journal of Engineering and Sustainable Development 15, no. 4 (2011): 205-226.

[5] van Lopik, Jan H., Roy Snoeijers, Teun CGW van Dooren, Amir Raoof, and Ruud J. Schotting. "The effect of grain size distribution on nonlinear flow behavior in sandy porous media." Transport in Porous Media 120, no. 1 (2017): 37 66. https://doi.org/10.1007/s11242-017-0903-3

[6] Sobieski, Wojciech, and Anna Trykozko. "Darcy's and Forchheimer's laws in practice-Part 1: The experiment." Technical Sciences/University of Warmia and Mazury in Olsztyn 17, no. 4 (2014): 321-335. 
[7] Hidayah, AH Nur, Md Nor Hasananb, and P. J. Ramadhansyahc. "Properties of Porous Blocks Using Different Sizes of Coarse Aggregate for Pavement." Journal of Advanced Research in Applied Mechanics 1, no. 1 (2014): 18-24.

[8] Razali, A. A., and A. Sadikin. "CFD simulation study on pressure drop and velocity across single flow microchannel heat sink." Journal of Advanced Research Design 8, no. 1 (2015): 12-21.

[9] Kumar, Prashant, and Frédéric Topin. "Predicting pressure drop in open-cell foams by adopting Forchheimer number." International Journal of Multiphase Flow 94 (2017): 123-136. https://doi.org/10.1016/i.ijmultiphaseflow.2017.04.010

[10] Batchelor, Cx K., and G. K. Batchelor. An introduction to fluid dynamics. Cambridge university press, 2000. https://doi.org/10.1017/CBO9780511800955

[11] Hlushkou, Dzmitry, and Ulrich Tallarek. "Transition from creeping via viscous-inertial to turbulent flow in fixed beds." Journal of Chromatography A 1126, no. 1-2 (2006): 70-85. https://doi.org/10.1016/i.chroma.2006.06.011

[12] Sochi, Taha. "Single-phase flow of non-Newtonian fluids in porous media." arXiv preprint arXiv:0907.2399 (2009).

[13] Fogler, H. Scott. Elements of Chemical Reaction Engineering. $4^{\text {th }}$ Ed., 2008.

[14] Quinn, Hubert M. "A reconciliation of packed column permeability data: Column permeability as a function of particle porosity." Journal of Materials 2014 (2014): 1-22. https://doi.org/10.1155/2014/636507

[15] Dias, Ricardo, José A. Teixeira, Manuel Mota, and Alexander Yelshin. "Tortuosity variation in a low density binary particulate bed." Separation and Purification Technology 51, no. 2 (2006): 180-184. https://doi.org/10.1016/i.seppur.2006.01.010 